\title{
Avaliacão de um programa universitário de ativídade física para idosos pelo modelo RE-AIM
}

\section{Evaluation of a university program of physical activity for aged by RE-AIM model}

Paula Fabricio Sandreschi ${ }^{1}$

Daniel Rogério Petreça $a^{1,2}$

Giovana Zarpellon Mazo’

\section{RESUMO}

O objetivo do presente estudo foi avaliar o programa de extensão universitária de atividades físicas para idosos desenvolvido pelo programa de extensão Grupo de Estudos da Terceira Idade (GETI). Para avaliação do GETI utilizou-se o modelo RE-AIM que é dividido em cinco dimensões: alcance, adoção, implementação, efetividade/eficácia e manutenção. Para a análise das dimensões do modelo RE-AIM foram utilizados: ficha diagnóstica para os idosos, os profissionais, bolsistas, voluntários e estagiários; também foi aplicada a bateria de testes Senior Fitness Test aos participantes. Fontes documentais de informações foram utilizadas para contemplar as análises das dimensões do RE-AIM. Os dados obtidos foram tratados por meio de estatística descritiva (frequência simples e relativa) e inferencial. Foi verificado um alcance de $1,5 \%$ do programa em nível municipal. A adoção a nível profissional docente foi de $21,9 \%$ e de nível discente de 14,2\%. Na implementação foi realizada adaptação do modelo lógico. $\mathrm{Na}$ efetividade/eficácia foi verificado que 91,7\% dos participantes perceberam melhorara na sua qualidade de vida e na resistência aeróbia durante um ano de intervenção. No entanto, houve piora da força de membros inferiores e manutenção das demais aptidões. Dos participantes, 52,5\% mantiveram-se praticantes durante as férias do programa. Conclui-se que o GETI possui alcance baixo, adoção suficiente e sua implementação se deu de forma estruturada e planejada. Na efetividade/eficácia, o programa cumpriu com seus objetivos propostos demonstrando manutenção devido à continuidade da prática durante o período de férias do programa.

\section{PALAVRAS-CHAVE}

Idoso; Atividade motora; Desenvolvimento de programas; Universidades.

\begin{abstract}
The goal of the present study was to assess the university extension program of physical activity for senior citizens developed by the extension program Grou of Studies of the Senior Age (GETI). For the assessment, GETI utilized the model RE-AIM that is divided in 5 dimensions: reach, adoption, implementation, effectivity/efficiency and maintenance. For the assessment of the dimensions of the model RE-AIM there were utilized: diagnostic file for the seniors, the professionals, the scholarship student, volunteers and interns; there was also applied the battery of test Senior Fitness Test on the participants. Documental sources of information were utilized to contemplate the dimensions assessments of the RE-AIM. The obtained data were treated by the descriptive statistics (simple and relative frequency) and inferential. There was verified a reach of $1,5 \%$ of the program on a municipal level. The adoption on the professional teaching level was 21,9\%, and on the students level $14,2 \%$. On the implementation was conducted the adaptation of the logical method. On the effectivity/efficiency was verified that $91,7 \%$ of the participants noticed an increase on their life quality and on their aerobic resistance during one year of intervention. However, there has been a decrease on the strength of their inferior members and maintenance of the other skills. Of the participants, $52,5 \%$ kept practicing during the program vacation. The conclusion is that the GETI has a low reach, enough adoption and it's implementation happened on a structured way and planed. on the effectivity/efficiency, the program fulfilled the proposed goals demonstrating maintenance due to the continuity of the practice on the vacation of the program.
\end{abstract}

\section{KEYWORDS}

Aged; Motor Activity; Program Development; Universities
Rev Bras Ativ Fís Saúde p. 270-283 DOI

http://dx.doi.org/10.12820/rbafs.v.20n3p270

1 Universidade do Estado de Santa Catarina - UDESC, Laboratório de Gerontologia, Florianópolis, Santa Catarina, Brasil.

2 Universidade do Contestado - UnC, Núcleo de Pesquisa em Saúde Coletiva e Meio Ambiente, Mafra, Santa Catarina, Brasil. 


\section{INTRODUCCÃO}

Observa-se, no Brasil, a Universidade como um dos principais agentes sociais empenhados em propor programas direcionados à população idosa por meio de atividades que promovam a saúde, o bem-estar psicológico e social e a cidadania dessa população, genericamente chamada de idosa ${ }^{1}$.

Nesse sentido, a dinâmica extensionista no Brasil está cada vez mais consagrada com os programas de promoção à saúde com ênfase em atividade física para idosos com a atuação de acadêmicos de Educação Física²

Esse é o caso do programa de extensão Grupo de Estudos da Terceira Idade (GETI), da Universidade do Estado de Santa Catarina (UDESC), que dentre suas ações, destacam-se os projetos de atividade física para idosos ${ }^{2}$. Apesar de ser um programa consolidado e reconhecido, ressalta-se que tão importante quanto colocar as estratégias de promoção à saúde em prática é a realização de avalições para que se constate se essas ações estão sendo efetivas em seus objetivos.

No fim da década de 1990, o pesquisador norte americano Dr. Russell Glasgow e sua equipe desenvolveram o modelo de avaliação RE-AIM, para auxiliar pesquisadores e gestores no planejamento e na avaliação de programas, buscando, desta forma, diminuir as lacunas entre a pesquisa e a prática e maximizar o impacto das intervenções na saúde pública ${ }^{3}$. O RE-AIM foi traduzido e adaptado culturalmente para a realidade brasileira por Almeida et al. ${ }^{4}$ e é constituído por cinco dimensões (Alcance, Adoção, Implementação, Efetividade/Eficácia e Manutenção).

Embora amplamente difundido internacionalmente, não são comuns estudos que utilizaram deste método no Brasil para avaliar programas de atividade física. No entanto, programas foram avaliados por meio de outras estratégias como entrevistas e questionários ${ }^{5}$. Conforme Benedetti et $\mathrm{al}^{6}$ esses meios de avaliação, apesar de importantes, acabam tornando-se restritos, não sendo possível mensurar seus reais efeitos na saúde pública; o que é reforçado por Kohl et al. ${ }^{7}$ o qual recomenda o desenvolvimento de intervenções efetivas como prioridade em saúde pública para aumentar o nível de atividade física da população.

Nesse contexto, o presente estudo justifica-se diante do envelhecimento populacional e da ampliação de implementações de programas voltados para a promoção da saúde com ênfase em atividade física e na escassez de avaliações realizadas de maneira multidimensional, que se faz necessária para estabelecer planejamento, implementação e sustentabilidade baseados em evidências. Além disso, este estudo pode contribuir para que sejam elaboradas estratégias em contextos semelhantes ao do presente estudo. Portanto, o objetivo do presente estudo foi avaliar um programa de extensão universitária de atividades físicas para idosos pelo Modelo RE-AIM.

\section{MÉTODOS}

\section{Tipo de estudo}

Este estudo foi realizado mediante a investigação descritiva com abordagem quantitativa e qualitativa dos dados. 


\section{Programa de Extensão Grupo de Estudos da Terceira Idade (GETI)}

O programa de extensão GETI do Centro de Ciências da Saúde e Esporte (CEFID) da Universidade do Estado de Santa Catarina (UDESC), criado em 1989 , tem dentre seus objetivos articular e integrar o ensino, a pesquisa e a extensão para melhorar a qualidade de vida do idoso cidadão ${ }^{2}$. Atualmente oferece, além de programas de cunho educativo e formativo, nove modalidades de atividade física, que foram analisadas no presente estudo.

\section{Participantes do estudo}

Todos os envolvidos com o GETI participaram do estudo, sendo 200 idosos, 8 profissionais, 5 bolsistas de extensão, 4 estagiários e 10 voluntários. O número de participantes variou de acordo com cada análise realizada no estudo.

\section{Instrumentos de dados}

Para avaliação do programa utilizou-se o modelo RE-AIM que foi desenvolvido, dentre outros objetivos, como um método para auxiliar no planejamento, na avaliação e na implementação de programas ${ }^{4}$. O modelo analisa as dimensões: Alcance, Adoção, Implementação, Efetividade/Eficácia e Manutenção (Quadro 1).

\begin{tabular}{|l|l|c|}
\hline Dimensões do RE-AIM & Definições & \multicolumn{2}{|c|}{ Nível de Avaliação } \\
\hline Alcance & $\begin{array}{l}\text { É o número absoluto, a proporção e a representatividade dos indivíduos que } \\
\text { estão dispostos a participar de uma determinada iniciativa comparada àqueles } \\
\text { que desistem ou àqueles potencialmente elegíveis. } \\
\text { É o número absoluto, a proporção e a representatividade das organizações e } \\
\text { dos agentes de intervenção que estão dispostos a iniciar um programa. }\end{array}$ & Individual \\
\hline Implementação & $\begin{array}{l}\text { No nível organizacional, refere-se à fidelidade dos agentes de intervenção aos } \\
\text { vários elementos de um protocolo de intervenção. Isso inclui a consistência no } \\
\text { fornecimento, conforme previsto, o tempo e o custo da intervenção. No nível } \\
\text { individual, refere-se à medida que os participantes utilizam as estratégias da } \\
\text { intervenção. }\end{array}$ & Individual e Organizacional \\
\hline Efetividade/eficácia & $\begin{array}{l}\text { É o impacto de uma intervenção sobre desfechos importantes incluindo } \\
\text { qualidade de vida, potenciais efeitos negativos e os resultados econômicos. }\end{array}$ & Individual \\
\hline Manutenção & $\begin{array}{l}\text { No nível organizacional, é à medida que um programa ou política torna-se } \\
\text { institucionalizado ou parte das práticas de rotina e políticas organizacionais. } \\
\text { No nível individual, a manutenção tem sido definida como os efeitos benéficos } \\
\text { em longo prazo (seis ou mais meses após o término da intervenção). }\end{array}$ & Individual e Organizacional \\
\hline
\end{tabular}

QUADRO 1 - Dimensões analisadas pelo modelo RE-AIM.

Adaptado de Almeida et al. ${ }^{4}$.

Para contemplar as informações necessárias para a análise de cada dimensão do RE-AIM, foram utilizadas diferentes informações, fontes e instrumentos que estão descritos abaixo:

\section{- Para os participantes idosos do GETI}

- Ficha diagnóstica: apresenta questões fechadas relacionadas aos dados sociodemográficos (local onde vive, idade, sexo e escolaridade) e auto percepção sobre melhoria da qualidade de vida (por meio da seguinte pergunta: Em geral, o senhor (a) diria que a sua qualidade de vida após o ingresso no GETI: melhorou, piorou ou ficou igual?); além de questões abertas referentes ao Programa (motivo de ingresso, forma de conhecimento e fatores adversos ocorridos relacionados ao GETI) e prática de atividade física (por 
meio da seguinte pergunta: $\mathrm{O}$ senhor (a) realiza atividade física durante as férias do Programa? Se sim, qual?) dos participantes.

- Bateria de testes (): mensura a aptidão física por meio de seis testes motores que avaliam a força dos membros superiores e inferiores, flexibilidade dos membros superiores e inferiores, agilidade/equilíbrio dinâmico e resistência aeróbia ${ }^{8}$.

\section{- Para os profissionais, bolsistas de extensão, estagiários e voluntários que atuam no GETI}

- Ficha diagnóstica: apresenta questões fechadas relacionadas aos dados sociodemográficos (idade, sexo, escolaridade e remuneração) e uma questão aberta referente ao Programa (motivo de ingresso no GETI).

Outras fontes de informação foram utilizadas no estudo, como dados fornecidos pelo Censo 2010 do Instituto Brasileiro de Geografia e Estatística ${ }^{9}$ sobre a população e o número de idosos residentes em Florianópolis, SC; o estudo epidemiológico EpiFloripa Idoso ${ }^{10}$ para verificar a proporção de pessoas com 60 anos ou mais e com ausência de dependência (isto é, não apresentar dependência de outra pessoa para a realização de atividades da vida diária) residentes em Florianópolis, SC (população considerada com características semelhantes com as dos participantes do programa); a Instrução Normativa $\mathrm{n}^{\circ}$ 01/2009 do GETI/CEFID/UDESC que apresenta informações sobre o funcionamento do programa, dentre eles critérios de participação neste e orientação dos bolsistas de extensão; documento com o número de bolsistas de extensão e de estudantes do CEFID e da extensão universitária do CEFID/ UDESC que foi fornecido pela Direção de Extensão do Centro; informações fornecidas pela Secretaria Acadêmica do CEFID sobre a quantidade de alunos e professores regularmente vinculados no curso de Educação Física; informação cedida pelo Setor Financeiro da UDESC sobre o processo de financiamento dos programas de extensão da universidade; dados concedidos pela secretaria do GETI sobre os não participantes entre o período de dezembro de 2013 a março de 2014. O Quadro 2 resume as informações, fontes ou instrumentos, forma de coleta de dados e população ou participantes do estudo, de acordo com as dimensões do RE-AIM.

\section{Procedimentos de coleta de dados}

O presente estudo foi aprovado pelo Comitê de Ética em Pesquisa com Seres Humanos da Universidade do Estado de Santa Catarina - UDESC sob número de protocolo 185/07.

Todos os instrumentos foram aplicados nos participantes durante as coletas de dados do GETI, que ocorreram no início e final de ano de 2013 (em março e dezembro) e no início do ano de 2014 (março), por estudantes universitários previamente treinados. Aqueles direcionados aos integrantes do GETI (profissionais, bolsistas, estagiários e voluntários)foram aplicados pelos pesquisadores do estudo em data e horário agendados.

\section{Tratamento dos dados}

Os dados obtidos foram tratados por meio de estatística descritiva (frequência absoluta e relativa) e inferencial (teste t para amostras pareadas para dados pa- 
ramétricos e teste de Wilcoxon para não paramétricos) que foram analisados no software estatístico SPSS® versão 20.0. As questões abertas foram interpretadas por meio de análise de conteúdo temática de acordo com Minayo ${ }^{11}$.

\begin{tabular}{|c|c|c|c|c|c|}
\hline & Informações & Fontes ou instrumentos & $\begin{array}{l}\text { Forma de coleta } \\
\text { de dados }\end{array}$ & $\begin{array}{l}\text { População ou participantes d } \\
\text { estudo }\end{array}$ & \\
\hline \multirow{4}{*}{ Alcance } & $\begin{array}{l}\text { Percentual de idosos em nível } \\
\text { municipal }\end{array}$ & Censo $2010^{10}$ & Documental & $\begin{array}{l}\text { Residentes em } \\
\text { Florianópolis }\end{array}$ & \\
\hline & $\begin{array}{l}\text { Percentual de idosos } \\
\text { independentes funcionalmente }\end{array}$ & Estudo EpiFloripa"11 & Documental & $\begin{array}{l}\text { Idosos residentes em } \\
\text { Florianópolis }\end{array}$ & \\
\hline & $\begin{array}{l}\text { Características sociodemográficas } \\
\text { dos idosos participantes }\end{array}$ & Ficha diagnóstica & Entrevista & Idosos participantes & \\
\hline & $\begin{array}{l}\text { Critérios de inclusão para entrar } \\
\text { no Programa }\end{array}$ & Normativa do GETI & Documental & - & \\
\hline \multirow{8}{*}{ Adoção } & Taxa de participação institucional & Normativa do GETI & Documental & - & \multirow{6}{*}{ NO } \\
\hline & $\begin{array}{l}\text { Taxa de participação dos } \\
\text { estudantes em relação ao } \\
\text { Programa }\end{array}$ & Secretaria acadêmica do CEFID & Documental & Acadêmicos do curso de EF & \\
\hline & $\begin{array}{l}\text { Taxa de participação de bolsistas } \\
\text { de extensão em relação ao } \\
\text { Programa }\end{array}$ & Direção de extensão do CEFID & Documental & $\begin{array}{l}\text { Bolsistas de extensão } \\
\text { estudantes de EF }\end{array}$ & \\
\hline & $\begin{array}{l}\text { Motivos de ingresso no Programa } \\
\text { dos bolsistas, estagiários e } \\
\text { voluntários }\end{array}$ & Ficha diagnóstica & Entrevista & Bolsistas integrantes & \\
\hline & Custos com recursos humanos & Ficha diagnóstica & Entrevista & Bolsistas integrantes & \\
\hline & $\begin{array}{l}\text { Nível de instrução dos } \\
\text { profissionais }\end{array}$ & Ficha diagnóstica & Entrevista & Profissionais & \\
\hline & $\begin{array}{l}\text { Motivos de ingresso no Programa } \\
\text { dos idosos }\end{array}$ & Ficha diagnóstica & Entrevista & Idosos participantes & \multirow[b]{2}{*}{$\mathrm{NI}$} \\
\hline & $\begin{array}{l}\text { Forma de conhecimento do } \\
\text { Programa pelos idosos }\end{array}$ & Ficha diagnóstica & Entrevista & Idosos participantes & \\
\hline \multirow{3}{*}{$\begin{array}{l}\text { Imple- } \\
\text { mentação }\end{array}$} & $\begin{array}{l}\text { Relação entre insumos, atividades } \\
\text { propostas e metas }\end{array}$ & Modelo lógico do GETI & Documental & & \\
\hline & $\begin{array}{l}\text { De que maneira os bolsistas são } \\
\text { orientados }\end{array}$ & Normativa do GETI & Documental & - & \\
\hline & Financiamento do Programa & Setor financeiro do CEFID & Documental & - & \\
\hline \multirow{3}{*}{ Efeti-vidade } & $\begin{array}{l}\text { Percepção da melhoria da } \\
\text { qualidade de vida após entrada no } \\
\text { programa }\end{array}$ & Ficha diagnóstica & Entrevista & Idosos participantes & \\
\hline & Nível de aptidão física & $\begin{array}{l}\text { Bateria de testes Senior Fitness } \\
\text { Test }\end{array}$ & $\begin{array}{l}\text { Aplicação dos } \\
\text { testes }\end{array}$ & Idosos participantes & \\
\hline & $\begin{array}{l}\text { Fatores adversos causados pela } \\
\text { prática de AF no GETI }\end{array}$ & Ficha diagnóstica & Entrevista & Idosos participantes & \\
\hline \multirow{4}{*}{$\begin{array}{l}\text { Manu- } \\
\text { tenção }\end{array}$} & $\begin{array}{l}\text { Motivos de desistência dos } \\
\text { bolsistas }\end{array}$ & Questionário & Entrevista & Ex bolsistas e voluntários & NO \\
\hline & $\begin{array}{l}\text { Prática de atividade física durante } \\
\text { as férias do Programa }\end{array}$ & Ficha diagnóstica & Entrevista & Idosos participantes & \multirow{3}{*}{$\mathrm{NI}$} \\
\hline & $\begin{array}{l}\text { Taxa de abandono ao longo dos } \\
\text { anos }\end{array}$ & Secretaria do GETI & Documental & - & \\
\hline & Motivos de desistência dos idosos & Questionário & Entrevista & Ex participantes do GETI & \\
\hline
\end{tabular}

QUADRO 2 - Informações, fontes ou instrumentos, forma de coleta de dados e população ou par-

ticipantes do estudo, de acordo com as dimensões do RE-AIM

Legenda: $\mathrm{EF}$ = educação física; $\mathrm{NO}$ = nível organizacional; $\mathrm{NI}$ = nível individual. 


\section{RESULTADOS}

A descrição dos resultados será feita de acordo com as cinco dimensões do modelo RE-AIM abordadas no presente estudo. O Quadro 3 apresenta uma síntese dos resultados.

\begin{tabular}{|c|c|c|}
\hline & Informações & Resultados \\
\hline & Critérios de inclusão para entrar no Programa & Ter idade acima de 55 anos e independência física \\
\hline \multirow{4}{*}{ Alcance } & Percentual de idosos em nível municipal & $11,4 \%(n=48.136)$ \\
\hline & Percentual de idosos independentes funcionalmente & $27,7 \%$ (n=13.371) não apresentam dependência física ${ }^{10}$ \\
\hline & Taxa de alcance em nível municipal & $1,5 \%$ \\
\hline & $\begin{array}{l}\text { Características sócio demográficas dos idosos } \\
\text { participantes }\end{array}$ & $\begin{array}{l}\text { Maioria são mulheres }(78,5 \%) \text {, são casados }(61,5 \%) \text {, tem ensino médio } \\
\text { completo ou escolaridade superior }(59,5 \%) \text { e possui uma renda mensal } \\
\text { acima de } 4 \text { salário mínimos }(52,5 \%)\end{array}$ \\
\hline \multirow{9}{*}{ Adoção } & Taxa de participação institucional & $100 \%$ por ser exclusivo em um Campus \\
\hline & Taxa de participação dos Professores & $\begin{array}{l}\text { Dos } 32 \text { professores efetivos, } 7 \text { coordenam projeto sendo a taxa de } \\
\text { participação de } 21,9 \%\end{array}$ \\
\hline & Taxa de participação dos Estudantes & $\begin{array}{l}\text { Dos } 466 \text { em 2013, } 18 \text { atuam no GETI, sendo a taxa de participação de } \\
3,8 \% \text {. }\end{array}$ \\
\hline & Taxa de participação de bolsistas de Extensão & $\begin{array}{l}\text { Dos } 28 \text { alunos que atuam na extensão do CEFID, } 4 \text { atuam no GETI, } \\
\text { sendo a taxa de participação de } 14,2 \% \text {. }\end{array}$ \\
\hline & $\begin{array}{l}\text { Motivos de ingresso no Programa dos bolsistas, } \\
\text { estagiários e voluntários }\end{array}$ & Contato com pessoas idosas 33\% seguido de vivência prática $20 \%$. \\
\hline & Custos com recursos humanos & Exposto na Figura 2. \\
\hline & Nível de instrução dos profissionais & $\begin{array}{l}\text { Professores doutores, doutorandos e mestrando na coordenação e } \\
\text { acadêmicos de educação Física na aplicação das atividades. }\end{array}$ \\
\hline & Motivos de ingresso no Programa dos idosos & $\begin{array}{l}33,5 \% \text { tem motivos relacionados à saúde, } 21,9 \% \text { incentivo de amigos, } \\
\text { parentes ou conhecidos }\end{array}$ \\
\hline & Forma de conhecimento do Programa pelos idosos & $65,9 \%$ conhecem o GETI por meio de amigo e parentes. \\
\hline \multirow{3}{*}{$\begin{array}{l}\text { Imple- } \\
\text { mentação }\end{array}$} & $\begin{array}{l}\text { Relação entre insumos, atividades propostas e } \\
\text { metas }\end{array}$ & Exposto na Figura 1 por meio do modelo lógico \\
\hline & De que maneira os bolsistas são orientados & $\begin{array}{l}\text { Orientados e supervisionados por uma coordenadora, } 7 \text { professores } \\
\text { universitários, } 3 \text { doutorandos e } 4 \text { mestrandos. }\end{array}$ \\
\hline & Financiamento do Programa & A fonte de fomento é a UDESC- atendimento é gratuito. \\
\hline \multirow{3}{*}{ Efetividade } & $\begin{array}{l}\text { Percepção da melhoria da qualidade de vida após } \\
\text { entrada no programa }\end{array}$ & $91,7 \%$ consideraram melhora na sua qualidade de vida. \\
\hline & Nível de aptidão física & $\begin{array}{l}\text { Diminuição da força de membros inferiores }(p=<0,01) \text {, aumento na } \\
\text { resistência aeróbia }(p=0,005) \text { e manutenção da força de membros } \\
\text { superiores }(p=0,155) \text {, flexibilidade de membros inferiores e superiores } \\
\text { - direito e esquerdo - ( } p=0,397 ; 0,932 ; 0,120 \text {, respectivamente), } \\
\text { agilidade e equilíbrio dinâmico }(p=0,241) \text {. }\end{array}$ \\
\hline & $\begin{array}{l}\text { Fatores adversos causados pela prática de AF no } \\
\text { GETI }\end{array}$ & $\begin{array}{l}22,7 \% \text { referiram alguma situação, sendo os mais frequentes } \\
\text { "desentendimento com colegas e/ou professores" e "acidente lquedas } \\
\text { ou mal estar) durante as aulas ou na ida ou volta à casa". }\end{array}$ \\
\hline \multirow{4}{*}{ Manutenção } & Motivos de desistência dos bolsistas & $\begin{array}{l}62,5 \% \text { interesse por conhecer outras áreas do curso seguido de } 25 \% \\
\text { pela incompatibilidade de horários }\end{array}$ \\
\hline & $\begin{array}{l}\text { Prática de atividade física durante as férias do } \\
\text { Programa }\end{array}$ & $52,5 \%$ afirmaram manter a $\mathrm{AF}$ nas férias. \\
\hline & Taxa de abandono ao longo dos anos & De 2013 para 2014 taxa de desistência de 5\% \\
\hline & Motivos de desistência dos idosos & $\begin{array}{l}60 \% \text { por problemas de saúde seguidos de } 30 \% \text { na perda do período de } \\
\text { matrícula }\end{array}$ \\
\hline
\end{tabular}

QUADRO 3 - Síntese dos resultados obtidos no presente estudo. 


\section{Alcance}

As atividades oferecidas no GETI são gratuitas, é necessário atender os seguintes critérios para participar do programa: possuir 55 anos ou mais de idade, ser fisicamente independente e apresentar atestado médico comprovando que está apto para a prática de exercício físico.

Não são necessárias estratégias de recrutamento de participantes, uma vez que a procura é maior do que a demanda oferecida. Sendo assim, é gerada uma lista de espera no início de cada ano e zerada no próximo, o número de pessoas dessa lista é em média de 50.

Cabe ressaltar que dos participantes que frequentam o GETI, 200 participam dos projetos de atividade física; desses, 35 moram em municípios próximos da região metropolitana de Florianópolis/SC e por isso não entraram no cálculo referente ao alcance.

Para tanto, realizou-se um levantamento entre os idosos residentes no município de Florianópolis/SC (população alvo) e aqueles que não possuem dependência física (elegíveis). Constatou-se que $11,4 \%(\mathrm{n}=48.136)$ da população municipal $(n=421.240)$ possuem 60 anos ou mais ${ }^{9}$; desses, $27,7 \%(n=13.371)$ não apresentam dependência física ${ }^{10}$. Portanto, estima-se que o alcance do GETI seja de 1,5\% em nível municipal $(\mathrm{n}=200)$.

Em relação às características sociodemográficas dos participantes das ações de atividade física no GETI em 2014, verificou-se que a média de idade geral é de $67(\mathrm{DP}=6,7)$ anos com idade mínima de 55 anos e idade máxima de 82 anos, que a maioria é mulher (78,5\%), casada (61,5\%), com ensino médio completo ou mais $(59,5 \%)$ e possui renda mensal familiar acima de quatro salários mínimos $(52,5 \%)$.

\section{Adoção}

Em nível organizacional, o GETI foi implantado somente no CEFID, portanto a participação institucional é de $100 \%$. Estão vinculados ao CEFID, atuando no Curso de Educação Física, 32 professores efetivos; desses, sete coordenam projetos de atividade física no GETI. Portanto a adoção profissional nível docente é de 21,9\%. Quanto à participação dos estudantes de Educação Física, são oferecidas bolsas de extensão. Constatou-se que dos 466 alunos matriculados em 2013 nos cursos de Educação Física oferecidos pela UDESC, 18 atuam nas atividades de extensão do GETI, sendo a taxa de participação de $3,8 \%$ (18/466). No entanto, ao considerar apenas os alunos estudantes de educação física e bolsistas de extensão $(n=28)$, quatro atuam no GETI, apresentando uma adoção profissional nível discente de 14,2\% (4/28).

Em relação aos bolsistas e voluntários de extensão do GETI e os motivos que os levaram a desenvolver seu trabalho no programa, destaca-se o contato com pessoas idosas $(n=5 ; 33 \%)$, seguido de uma vivência prática da teoria aprendida durante as aulas da graduação $(n=3 ; 20 \%)$, a oportunidade do estágio obrigatório em Atividade Física Adaptada ( $\mathrm{n}=2 ; 13 \%)$, o contato com a pesquisa $(n=1 ; 7 \%)$ e o interesse por atividades complementares $(n=1 ; 7 \%)$, três $(20 \%)$ não responderam.

Em nível individual, referente aos participantes do programa, constatou-se que os motivos que os levaram a ingressar no GETI foram principalmente relacionados à saúde $(33,5 \%)$ seguido do incentivo de amigos, parentes ou conhecidos $(21,9 \%)$. Outros motivos também foram citados, como o gosto 
pela prática de atividade física $(14,3 \%)$, sociabilização $(11,6 \%)$, busca pela melhora da qualidade de vida $(5,8 \%)$, proximidade de casa $(4,9 \%)$, credibilidade $(3,1 \%)$, ocupação do tempo $(2,7 \%)$, gratuidade $(1,8 \%)$ e praticar atividade física com orientação $(0,4 \%)$.

Além dos motivos de ingresso, contatou-se que a maioria conheceu o GETI por meio de amigos ou parentes $(65,9 \%)$. No entanto, outros meios foram citados, como indicação da própria Universidade (UDESC/CEFID) $(13,2 \%)$ e de médicos (7,3\%).

\section{Implementação}

Com o intuito de expor as relações existentes entre os insumos, as atividades propostas, as metas a serem alcançadas e os resultados esperados a Figura 1 apresenta o modelo lógico do GETI nas ações de atividade física. Ao longo dos anos o programa passou por alterações (em número de alunos, projetos, profissionais, colaboradores e parcerias) e no formato atual possui nove projetos de atividade física.

FIGURA 1 - Modelo lógico de funcionamento dos projetos de Atividade Física do GETI.

Melhorar a qualidade de vida do idoso cidadão, por meio da articulação e integração do ensino, pesquisa e a extensão na comunidade universitária.

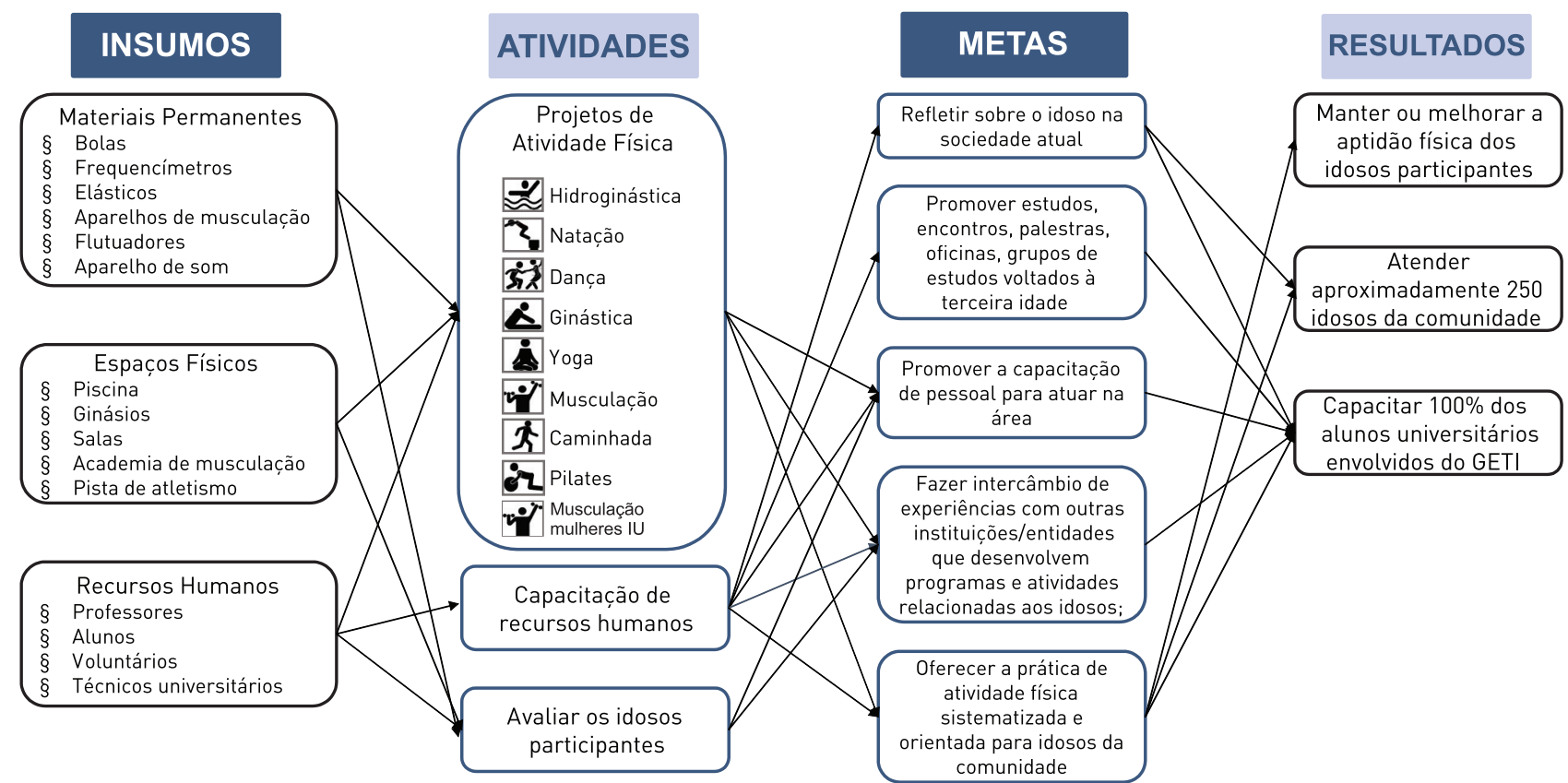

As aulas são ministradas por bolsistas e voluntários de extensão do programa que são orientados e supervisionados por uma coordenadora, professores universitários $(n=7)$, alunos de mestrado $(n=4)$ e doutorado $(n=3)$, profissionais de Educação Física e acontecem no período vespertino, tem duração média de 60 minutos e ocorrem duas vezes por semana, exceto yoga (1 vez) e musculação (3 vezes).

A fonte de fomento para a implementação e funcionamento dos projetos é a UDESC e por isso o atendimento é gratuito. O CEFID disponibiliza os espaços físicos necessários para que as atividades aconteçam e a Pró-Reitoria de 
extensão subsidia os recursos para a concessão de bolsas, além de destinar parte da carga horária dos professores para que atuem nestas ações. Os materiais permanentes e de consumo necessários são oriundos de solicitações ao setor administrativo ou de editais provenientes do governo estadual ou federal, esses valores variam de acordo com o ano. Portanto, o GETI caracteriza-se como um programa público.

Quanto à remuneração destinada e à carga-horária disponibilizadas pelos recursos humanos, a coordenadora do programa $(n=1)$ possui 40 horas/ mês destinadas e remuneração de $\mathrm{R} \$$ 93,00/hora, os professores responsáveis $(\mathrm{n}=7)$ pelas modalidades de atividade física designam 15 horas/mês com remuneração de $\mathrm{R} \$$ 60,13/hora; os doutorandos (n=3), 8h/mês e $\mathrm{R} \$ 13,70 /$ hora; os mestrandos ( $n=4), 8$ horas/mês e R $\$ 9,37 /$ hora; os bolsistas de extensão $(n=3)$ e apoio discente $(n=2), 80$ horas/mês e $\mathrm{R} \$ 5 /$ hora; os estagiários $(n=4) 80$ horas/ mês e os voluntários $(\mathrm{n}=10), 8$ horas/mês e ambos não recebem remuneração. Para fins de comparação ao longo do tempo, cabe ressaltar que o valor do Salário Mínimo, no momento da coleta de dados era de $\mathrm{R} \$ 678,00$.

Por ser um programa universitário e utilizar o espaço físico e recursos humanos da UDESC, o GETI tem seu período de funcionamento (fevereiro a dezembro) de acordo com o calendário acadêmico proposto pela instituição.

\section{Efetividade/ Eficácia}

Com relação à efetividade, foi verificado que o principal objetivo do GETI vem sendo alcançado. Ao questionar 157 participantes do programa durante o ano de 2013, constatou-se que a maioria (91,7\%), considerou melhora na sua qualidade de vida após o início da prática de atividade física nos projetos oferecidos. No entanto, $5,7 \%$ relataram que sua qualidade de vida se manteve igual e 2,5\% que a mesma piorou após o ingresso.

Além deste objetivo, o GETI visa melhorar e/ou manter a aptidão física dos seus participantes. Para tanto, ao serem avaliados quanto à aptidão física $(\mathrm{n}=200)$ - por meio da bateria no início e no final do ano de 2013 apresentaram os seguintes resultados, descritos na Tabela 2.

TABELA 1 - Comparação entre as médias dos resultados obtidos nos testes de aptidão física da Senior Fitness Test no início e no final do ano de 2013 pelos praticantes de atividade física no GETI.

\begin{tabular}{lccc}
\hline Testes (n=133) & $\begin{array}{c}\text { Início } \\
\text { Média (DP) }\end{array}$ & $\begin{array}{c}\text { Final } \\
\text { Média (DP) }\end{array}$ & $p$ \\
\hline Força de membros inferiores $^{*}$ & $13,6(4,0)$ & $12,0(3,6)$ & $<0,0)^{*}$ \\
\hline Força de membros superiores $^{*}$ & $16,3(6,6)$ & $15,2(4,4)$ & 0,155 \\
\hline Flexibilidade de membros inferiores $^{\mathrm{cm}}$ & $2,0(12,2)$ & $1,7(7,7)$ & 0,397 \\
\hline Flexibilidade de membro superior $(\mathrm{d}) \mathrm{cm}^{\mathrm{cm}}$ & $-6,7(9,4)$ & $-7,6(12,1)$ & 0,932 \\
\hline Flexibilidade de membro superior $(\mathrm{e}) \mathrm{cm}^{\mathrm{c}}$ & $-10,0(10,9)$ & $-12(11,5)$ & 0,120 \\
\hline Agilidade e equilíbrio dinâmico $^{\mathrm{s}}$ & $6,0(1,7)$ & $5,8(1,7)$ & 0,241 \\
\hline Resistência aeróbia $^{\mathrm{m}}$ & $449,0(198,0)$ & $493,0(167,1)$ & $0,005^{*}$ \\
\hline
\end{tabular}

$*=p<0,05 ; Z$ = valor do teste Wilcoxon; DP = Desvio padrão; $(d)$ = lado direito; (e) = lado esquerdo; $¥=$ Repetições; $\mathrm{cm}=$ centímetros; $\mathrm{s}=$ segundos; $\mathrm{m}=$ metros.

Houve diminuição significativa na força de membros inferiores o que demonstra decréscimo dessa capacidade física no decorrer do ano. Foi verificado, também, aumento significativo na resistência aeróbia; demonstrando melhora 
desta capacidade física. Quanto às outras aptidões - flexibilidade de membros inferiores e superiores, agilidade e equilíbrio dinâmico e força de membros inferiores - não apresentaram diferença, verificando manutenção dessas.

Ao verificar a ocorrência de fatores adversos (quedas, acidentes, desentendimentos, entre outros) ocorridos na participação do programa foram entrevistados 198 participantes em 2013, sendo que, a maioria (77,2\%) relatou nunca ter sofrido esse tipo de circunstância com a prática das atividades; $22,7 \%$ referiram alguma situação. Os outros fatores com menor frequência foram "desentendimento com colegas e/ou professores" ( $n=10)$ e "acidente (quedas ou mal estar) durante as aulas ou na ida ou volta a casa" $(\mathrm{n}=8)$.

\section{Manutenção}

Quanto à manutenção em nível organizacional; o motivo mais citado para a desistência dos alunos de Educação Física (ex-bolsistas) do GETI foi o interesse por conhecer outras áreas do curso $(n=5 ; 62,5 \%)$, seguindo da incompatibilidade de horários com outras atividades $(\mathrm{n}=2 ; 25 \%)$ e a conclusão do curso de graduação $(\mathrm{n}=1 ; 12,5 \%)$.

Tratando-se da manutenção em nível individual, os participantes quando questionados quanto à manutenção da prática de atividades físicas durante as férias, 52,5\% em 2014, afirmaram manter a prática regular neste período.

Em relação à desistência, apenas 10 participantes não retornaram ao programa de um ano (2013) para o outro (2014); portanto a taxa de desistência foi de $5 \%$, sendo que os motivos foram: problemas de saúde $(\mathrm{n}=6 ; 60 \%)$, perda do período de matrícula $(n=3 ; 30 \%)$ e incompatibilidade de horário $(n=1 ; 10 \%)$.

\section{DISCUSSÃO}

Para o presente estudo, foi utilizado o Modelo RE-AIM para avaliação dos projetos de atividade física do Programa de extensão universitária Grupo de Estudos da Terceira Idade da Universidade do Estado de Santa Catarina.

Observa-se que o baixo alcance do GETI $(1,5 \%)$ de participantes em nível municipal se deve ao caráter extensionista do programa, visto que, a universidade não tem a pretensão de atender a população em geral, pois seu enfoque é na formação de recursos humanos. Segundo o Plano Nacional de Extensão ${ }^{12}$, a extensão universitária deve seguir a premissa de buscar o equilíbrio entre as demandas socialmente exigidas e as inovações que surgem com o trabalho acadêmico, além de criar condições para a participação da universidade na elaboração de políticas públicas voltadas para a população.

Quanto ao perfil sociodemográfico, observa-se que os idosos do GETI apresentam um nível escolaridade e renda elevados. Estudo sobre o perfil dos idosos de Florianópolis constatou que 37,4\% tem ensino médio completo ou mais e 24,8\% renda mensal familiar acima de quatro salários mínimos ${ }^{13}$. Vale destacar que o percentual do GETI e de Florianópolis quanto à escolaridade e renda é superior ao nacional, $17,4 \%$ e $22,9 \%$ (acima de 2 salários mínimos), respectivamente 9

Diante desses resultados, cabe ao programa refletir sobre os critérios para inclusão dos participantes. Ou seja, priorizar o atendimento à indivíduos em situação de vulnerabilidade social.

Quanto à distribuição dos idosos participantes do GETI pelo município de Florianópolis/SC, constatou-se que a maior parte deles reside no mes- 
mo bairro ou em próximos de onde ocorrem as atividades. As mesmas são oferecidas pelo CEFID que localiza-se no bairro Coqueiros, pertencente à área continental do município. O bairro é classificado como o quinto que mais possui idosos ${ }^{9}$. Devido à grande concentração de idosos residindo nesse bairro de Florianópolis há uma grande procura dessa população pelas atividades do GETI, não necessitando de divulgação do programa. O GETI possui um número de vagas que são supridas pela grande demanda e que geralmente resulta em uma lista de espera para que idosos ingressem, para que isso ocorra não é realizada nenhuma triagem baseada no poder aquisitivo desses.

Estudos apontam que os idosos da região continental de Florianópolis são mais ativos fisicamente quando comparados com outros de outras regiões do município ${ }^{14,15}$. Observa-se, portanto, que o programa pode estar influenciando nesse nível de atividade física.

Quanto à adoção profissional nível discente, constatou-se que a taxa de participação de alunos do curso de Educação Física é pequena. O edital para concessão de bolsas lançado pela UDESC em 2014 define o número de bolsas de extensão conforme a posição do programa no e limita no máximo a três bolsas de extensão de 20 horas semanais cada, que podem ser convertidas em seis bolsas de 10 horas semanais ${ }^{16}$. Esse limite de bolsas pode ser um fator que impede maior participação discente no GETI.

Outro fator que pode ser limitador para a participação discente, é o interesse por outras áreas de atuação, se não com idosos; resultado apresentado na manutenção nível organizacional. Isso pode ser reflexo da oportunidade que os alunos têm de participar de outras ações e programas de extensão no CEFID. O Centro conta atualmente com 49 ações de extensão distribuídas em 12 Programas e em quatro principais eixos temáticos: atividade física e saúde, iniciação esportiva e aperfeiçoamento, idosos e pessoas com deficiências ${ }^{17}$.

Os bolsistas de extensão relataram que o contato com pessoas idosas foi o principal motivo de ingresso no Programa. Diante disso, observa-se a necessidade de vivência prática no trabalho com a atividade física para idoso a fim de melhorar a formação acadêmica dos alunos.

Em relação aos participantes do GETI o principal motivo de ingresso no programa foi relacionado à saúde. De modo geral, os idosos buscam programas de atividade física por esse motivo ${ }^{18}$. Essa preocupação pode ser causada pela maior probabilidade de ocorrência de doenças crônicas e consequentes incapacidades associadas ao envelhecimento ${ }^{19}$, caracterizando a prática como fator protetor ou tratamento não farmacológico para esses declínios.

A implementação de programas para idosos é incentivada por diferentes órgãos oficiais que trabalham na promoção de saúde e estão sendo implantados em diversos municípios ${ }^{20}$. Muitas vezes ocorrem por iniciativa de universidades por meio da extensão universitária, que em alguns casos une-se ao ensino e à pesquisa ${ }^{15}$, caso do programa avaliado nesse estudo.

Diante desse processo, a implementação do GETI tem características semelhantes aos outros programas universitários para idosos (insumos, modalidades, recursos humanos, frequência, duração, intensidade ${ }^{20}$.

De acordo com o modelo lógico apresentado, a implementação do GETI está sendo executada quase em sua totalidade em relação aos insumos, atividades, metas e resultados. Apesar disso, um fator limitador quanto à implementação é o cálculo dos custos totais, pois gastos e manutenção com infra- 
estrutura são de difíceis acessos e pouco divulgados em serviços públicos, o que limitou essa variável, ficando restrito apenas aos valores de hora/aula dos recursos humanos.

Com relação à efetividade, os sujeitos participantes do GETI referiram melhoria na sua qualidade de vida após a entrada no programa. Autores apontam para resultados positivos com relação à influência de programas de atividade física para idosos na qualidade de vida ${ }^{21}$. Além disso, o programa tem como meta a melhora ou manutenção da aptidão física. Foi verificado que houve diminuição significativa na força de membros inferiores. A partir da quinta década de vida pode ocorrer redução em torno de 8 a $15 \%$ na força ${ }^{22}$; ainda é visto uma prevalência da diminuição dessa valência nos membros inferiores quando comparados aos membros superiores no processo de envelhecimento ${ }^{23}$. No entanto, é necessário que a partir desse resultado sejam criadas estratégias para o desenvolvimento dessa aptidão física para que seu decréscimo natural seja minimizado. Isso pode ser realizado com mais ênfase na capacitação dos bolsistas e na supervisão; dessa forma, havendo alterações no planejamento e execução das aulas.

Constatou-se também o aumento significativo na resistência aeróbia. Estudos de intervenção têm demonstrado que geralmente adultos mais velhos que completam um programa de atividade física produzem aumentos significativos na capacidade cardiorrespiratória ${ }^{24}$ além de desempenhar papel importante na melhora do desempenho cognitivo ${ }^{27}$.

Para as demais capacidades físicas (flexibilidade de membros inferiores e superiores, força de membros superiores e agilidade/equilíbrio dinâmico) avaliadas não houve diferença significativa entre os testes realizados no começo e no final do ano, demonstrando manutenção das mesmas ao longo do ano, fator fundamental segundo Chodzko-Zajko ${ }^{25}$ para que ocorra sustentação da independência física.

Com relação à manutenção em nível individual, a maioria dos participantes entrevistados em 2014 afirma manter o exercício regularmente durante as férias do GETI. Eiras et al ${ }^{19}$ também constaram que a grande parte dos entrevistados mantiveram a prática nas férias do programa. Diversos podem ser os fatores que influenciam a manutenção da prática de exercício físico, entre eles, o bem-estar ${ }^{19}$ e o gosto pela atividade realizada ${ }^{26}$, entre outros. Estes fatores podem contribuir com a manutenção de exercícios durante as férias.

Constatou-se que a taxa de desistência foi de 5\%. Quando comparado com outro estudo que relatou a taxa de desistência de idosos participantes de um programa universitário de atividade física do Rio Grande do Sul o qual apresentou um percentual de $31 \%{ }^{28}$; confirma-se que o número de desistentes do GETI pode ser considerado baixo. Os participantes desistentes do programa, entre 2013 e 2014, relataram como principal motivo problemas de saúde o que é confirmado pelos estudos de Cardoso et al. ${ }^{26}$. Isso pode ser motivado pelo declínio dos processos cognitivos e da funcionalidade motora comuns no envelhecimento que influenciam na execução de tarefas funcionais diárias e as doenças ${ }^{29}$.

Apesar das amplas contribuições atribuídas ao GETI e para o planejamento de futuras intervenções voltadas para a promoção de atividade física dos idosos, o presente estudo apresenta algumas limitações que devem ser supridas em outros estudos, como: avaliação dos participantes quanto à qualidade de vida de forma longitudinal (pré e pós intervenção) e por meio de um ins- 
trumento validado, além da aptidão física que não foi avaliada de acordo com as diferentes modalidades de exercícios físicos, o que pode ter influenciado as variáveis referentes à efetividade; além disso, não foi efetuada análise de um grupo controle e nem foi relatada a qualidade psicométrica dos instrumentos. $\mathrm{O}$ alcance foi calculado com dados de estudos que tiveram como sujeitos pessoas com 60 anos ou mais, isso aconteceu por falta de informações epidemiológicas de pessoas com 55 anos ou mais, como os participantes do GETI.

O GETI em nível municipal apresentou baixo alcance em termos de participantes, mas por ser um programa de extensão universitária e por seus objetivos considera-se um alcance satisfatório. No entanto, não há critério claro na literatura para qualificar o alcance de programas; portanto, cada contexto precisa ser avaliado especificamente.

Com relação à adoção, em nível profissional a mesma demonstrou ser suficiente para que todas as atividades sejam executadas. Já a adoção individual pode ser considerada alta, visto que recrutamentos de participantes não são necessários para que haja preenchimento das vagas oferecidas. Quanto à implementação, a mesma se deu de forma estruturada e planejada, o que reflete na constatação de efetividade/eficácia do programa, o qual comprovou cumprir seus objetivos propostos, apesar da piora da força dos participantes no decorrer do ano. Além disso, a manutenção demonstrou ser efetiva por razão dos participantes continuarem praticando atividade física durante as férias e a taxa de desistência do programa ser baixa.

Os resultados do presente estudo auxiliarão no planejamento e gestão das ações desenvolvidas pelo GETI, no que diz respeito aos participantes, professores responsáveis e alunos envolvidos, bem como, torna-se um parâmetro para a implementação de outros programas.

\section{REFERÊNCIAS}

1. Veras RP, Caldas CP. Promovendo a cidadania e a saúde do idoso: o movimento das universidades da idoso. Ciênc Saúde Colet. 2004; 9:423-32.

2. Mazo GZ, Cardoso AS, Dias RG, Balbé, GP, Virtuoso JF. Do diagnóstico à ação: Grupo de Estudos da Idoso: Alternativa para a promoção do envelhecimento ativo. Rev Bras Ativ Fis Saúde. 2009; 14(1):65-70.

3. Glasgow TM, Vogt SMB. Evoluating the public health impact of health promotion interventions: the RE-AIM framework. Am J Public Health. 1999; 89(9):1322-27.

4. Almeida FA, Brito FA, Estarbrooks PA. Modelo RE-AIM: tradução e adaptação cultural para o Brasil. REFACS. 2013; 1(1):6-16.

5. Reis RS, Hallal PC, Parra DC, Ribeiro IC, Brownson RC, Pratt M, et al. Promoting physical activity through community-wide policies and planning: findings from Curitiba, Brazil. J. Phys Act Health. 2010; 7(2):137-45.

6. Benedetti TRB, Meurer ST, Schwignel A, Chodzko-zajko W, Brito FA, Almeida FA. RE-AIM: uma proposta de avaliação de programas de atividade física. Kairós. 2014; 17(2):295-314.

7. Kohl HW, Craig CL, Lambert EV, Inoue S, Alkandari JR, Leetongin G. et al. The pandemic of physical inactivity: global action for public health. Lancet. 2012; 380:67-78.

8. Rikli R, Jones C. Development and validation of a functional fitness test for community residing older adults. J Aging Phys. Act. 1999; 7(2):129-61.

9. Instituto Brasileiro de Geografia e Estatística - IBGE. Síntese de indicadores sociais: uma análise das condições de vida da população brasileira; 2010. Rio de Janeiro: 2010.

10. Giehl MWC, Schneider IJC, Corseuil HX, Benedetti TRB, D’orsi E. Atividade física e percepção do ambiente em idosos: estudo populacional em Florianópolis. Rev Saúde Pública. 2012; 46(3):516-25.

11. Minayo MCS. O desafio do conhecimento: pesquisa qualitativa em saúde. São Paulo: Hucitec, 2008. 
12. Brasil. Plano Nacional de Extensão Universitária. Fórum de Pró-Reitores de Extensão das Universidades Públicas Brasileiras e SESu / MEC. 2011.

13. Medeiros FL, Xavier AJ, Schneider IJC, Ramos LR, d'Orsi E. Inclusão digital e capacidade funcional de idosos residentes em Florianópolis, Santa Catarina, Brasil (EpiFloripa 2009-2010). Rev Bras Epidemiol. 2012; 15(1):106-22.

14. Huhnen AP, Lopes MA, Benedetti TRB. Recursos econômicos e atividades físicas de idosos de Florianópolis-SC. Rev Bras Ativ Fis Saúde. 2006; 11(2).

15. Mazo GZ, et al. Grupo de Estudos da Terceira Idade-GETI: uma proposta de integração entre extensão, ensino e pesquisa voltados à pessoa idosa. Revista Conexão UEPG. 2013; 9(1):94-105.

16. Universidade do Estado de Santa Catarina. Centro de Ciências da Saúde e do Esporte. Direção de extensão: Catálogo das Ações de Extensão CEFID/UDESC. Florianópolis: Ed. da UDESC, 2014.

17. Universidade do Estado de Santa Catarina. Programa de Apoio à extensão - PAEX. Edital PAEX 05/2013. Florianópolis. 2013.

18. Eiras SB, Silva WHA, Souza DL, Vendruscolo R. Fatores de adesão e manutenção da prática de atividade física por parte de idosos. Rev Bras Ciênc Esporte. 2010; 31(2):75-89.

19. Rosa TEC, Benício MHD, Latorre MRDO, Ramos LR. Fatores determinantes da capacidade funcional entre idosos. Rev Saúde Pública. 2003; 37(1):40-8.

20. Benedetti TRB, Gonçalves LHT, Mota JAPS. Uma proposta de política pública de atividade física para idosos. Texto Contexto Enferm. 2007; 16(3).

21. Silva MF, G NBA, Lanferdini FJ, Marcon M, Dias CP. Relação entre os níveis de atividade física e qualidade de vida de idosos sedentários e fisicamente ativos. Rev Bras Geriatr Gerontol 2012; 15(4):635-42.

22. Deschenes MR. Effects of aging on muscle fibre type and size. Sports Medicine. 2004; (12):809-824.

23. Hughes VA, Fronteira WR, Wood M, Evans WJ, Dallal GE, Roubenoff R, et al. Longitudinal muscle strength changes in older adults: influence of muscle mass, physical activity and health. J Gerontol A Biol Sci Med Sci. 2001; 56(5):209-17.

24. Bherer L, Erickson KI, Lui-ambrose TA. Review of the Effects of Physical Activity and Exercise on Cognitive and Brain Functions in Older Adults. J Aging Research. 2013.

25. Chodzko-zajko WJ. ACSM's exercise for older adults. Philadelphia: WoltersKluwer/ Lippincott Williams \& Wilkins, 2014.

26. Cardoso AS, Borges LJ, Mazo GZ, Benedetti TB, Kuhnen AP. Fatores influentes na desistência de idosos em um programa de exercício físico. Movimento. 2008; 15(4):225-39.

27. Rogers CE, Cordeiro M, Perryman E. Maintenance of Physical Function in Frail Older Adults. Nurs Clin N Am. 2014; 49(2):147-56.

28. Hauser E, Gonçalves AK, Martins VF, Blessmann EJ. Motivos de desistência em um programa de atividades física para idosos. Kairós. 2014; 17(2): 43-56.

29. Spirduso WW. Dimensões Físicas do Envelhecimento. Tradução: Paula Bernardi. Barueri: Manole. 2005.

ENDEREÇO PARA

CORRESPONDÊNCIA

PAULA FABRICIO SANDRESCHI

Rua Pascoal Simone, 358, 88080-350,

(48) 3321-8611

RECEBIDO 21/01/2015

E-mail: paula.sandreskifagmail.com 